\title{
ORTHODOX T-SEMIGROUPS
}

\author{
M.K. SEN \\ Department of Pure Mathematics \\ 35, Ballygunge Circular Road \\ Calcutta - 700019 , India \\ N.K. SAHA \\ Department of Mathematics \\ Pingla Thana Mahavidyalaya \\ P.0. Maligram, Dist. Midnapore \\ Pin - 721 140, India \\ (Received December 7, 1988)
}

\begin{abstract}
Let $M=\{a, b, c, \ldots\}$ and $\Gamma=\{\alpha, \beta, \gamma, \ldots\}$ be two non-empty sets. $M$ is called a $\Gamma$-semigroup if $a a b \varepsilon M$, for $\alpha \varepsilon \Gamma$ and $b \varepsilon M$ and $(a a b) B c=a \alpha(b B c)$, for all $a, b, c \in M$ and for $a l l \alpha, \beta \varepsilon \Gamma$. A semigroup can be considered as a $\Gamma$-semigroup. In this paper we introduce orthodox $\Gamma$-semigroups and extend different results of orthodox semigroups to orthodox r-semigroups.
\end{abstract}

KEY WORDS AND PHRASES. Semigroup, $\Gamma$-semigroup, Orthodox $\Gamma$-semigroup, Inverse Г-semigroup .

1980 AMS SUBJECT CLASSIFICATION CODE. 20M.

1. INTRODUCTION.

Let $A$ and $B$ be two non-empty sets, $M$ the set of all mappings from $A$ to $B$, and $\Gamma$ a set of some mappings from $B$ to $A$. The usual mapping product of two elements of $M$ can not be defined. But if we take $f, g$ from $M$ and $\alpha$ from $r$ then the usual mapping product $f \circ g$ can be defined. Also we find that $f \circ \varepsilon M$ and $(f \circ g)$ bh $=f \alpha(g$ bh) for $f, g, h \in M$ and $\alpha, \beta \in \Gamma$.

If $M$ is the set of mxn matrices and $\Gamma$ is a set of some nxm matrices over the field of real numbers, then we can define $A_{m, n} \alpha_{n, m}{ }_{m, n}$ such that

$\left(A_{m, n} \alpha_{n, m}^{B}{ }_{m, n}\right) B_{n, m} C_{m, n}=A_{m, n} \alpha_{n, m}\left(B_{m, n}{ }_{n, m} C_{m, n}\right)$ where $A_{m, n}, B_{m, n}, C_{m, n} \in M$ and

$\alpha_{n, m}, \beta_{n, m} \varepsilon \Gamma$. An algebraic system satisfying the associative property of the above type is a r-semigroup (Saha [1]).

DEFINITION 1.1. Let $M=\{a, b, c, \ldots\}$ and $\Gamma=\{a, \beta, \ldots\}$ be two non-empty sets. $M$ is called a $\Gamma$-semigroup if (i) $a \alpha b \varepsilon M$ for $\alpha \varepsilon \Gamma$ and $a, b \varepsilon M$ and (ii) ( $a$ ab) $B c=a \alpha(b B c)$, for $a l 1 a, b, c \in M$ and for all $\alpha, \beta \varepsilon \Gamma$. 
A semigroup can be considered a r-semigroup in the following sense. Let $S$ be an arbitrary semigroup. Let 1 be a symbol not representing any element of $s$. Let us extend the given binary relation in $S$ to $S U 1$ by defining $11=1$ and $1 a=a l=a$ for all a in $\mathrm{S}$. It can be shown that $\mathrm{S} U 1$ is a semigroup with identity element 1.

Let $\Gamma=\{1\}$. Putting $a b=a l b$ it can be shown that the semigroup $S$ is $a$ $\Gamma$-semigroup where $\Gamma=\{1\}$. Since every semigroup is a $\Gamma$-semigroup in the above sense, the main thrust of our work is to extend different fundamental results of semigroups to $\Gamma$-semigroups. In Sen and Saha [2] and Saha [1,3,4] we have extended some results of semigroups to $\Gamma$-semigroups. In this paper we want to introduce orthodox $\Gamma$-semigroups and we want to extend results of Hall [5] and Yamada [6] to r-semigroups.

\section{PRELIMINARIES.}

We recall the following definitions and results from [1], [2], [3] and [4].

DEFINITION 2.1. Let $M$ be a $\Gamma$-semigroup. A non-empty subset $B$ of $M$ is sald to be a $\Gamma$-subsemigroup of $M$ if $B \Gamma B \subset B$.

DEFINITION 2.2. Let $M$ be a $\Gamma$-semigroup. An element a $\varepsilon M$ is said to be regular if a $\varepsilon$ a $M \Gamma a$, where $a \Gamma M \Gamma a=\{a a b \beta a: b \varepsilon M, \alpha, \beta \varepsilon \Gamma\} . \quad A \Gamma$-semigroup $M$ is said to be regular if every element of $M$ is regular.

EXAMPLE 2.1. Let $M$ be the set of $3 \times 2$ matrices and $\Gamma$ be a set of some $2 \times 3$ matrices over a field. We show that $M$ is a regular $\Gamma$-semigroup. Let $A \varepsilon M$, where $A=\left(\begin{array}{ll}a & b \\ c & d \\ e & f\end{array}\right)$ Then we choose $B \varepsilon \Gamma$ according to the following cases such that $A B A B A=A B A=A$.

CASE 1. When the submatrix $\left(\begin{array}{ll}a & b \\ c & d\end{array}\right)$ is non-singular, then ad - bc $\neq 0$. e,f may both be 0 or one of them is 0 or both of them are non-zero.

Then $B=\left(\begin{array}{lll}\frac{d}{a d-b c} & \frac{-b}{a d-b c} & 0 \\ \frac{-c}{a d-b c} & \frac{a}{a d-b c} & 0\end{array}\right)$ and we find $A B A=A$.

CASE 2. af- be $\neq 0$. Then $B=\left(\begin{array}{ccc}\frac{f}{a f-b e} & 0 & \frac{-b}{a f-b e} \\ \frac{-e}{a f-b e} & 0 & \frac{a}{a f-b e}\end{array}\right)$ and $A B A=A$.

CASE 3. cf - de $\neq 0$. Then $B=\left(\begin{array}{lll}0 & \frac{f}{c f-d e} & \frac{-d}{c f-d e} \\ 0 & \frac{-e}{c f-d e} & \frac{c}{c f-d e}\end{array}\right)$ and $A B A=A$.

CASE 4. When the submatrices are singular. Then either $\{a d-b c=0$ or $\left\{\begin{array}{l}\text { ad }-\mathrm{bc}=0 \\ \mathrm{af}-\mathrm{de}=0\end{array}\right.$ 
If all the elements of $A$ are 0 , then the case is trivial. Next we consider at least one of the elements of $A$ is non-zero, say $a_{i j} \neq 0,1=1,2,3$ and $j=1,2$. Then we take the $b_{j i}$ th element of $B$ as $\left(a_{i j}\right)^{-1}$ and the other elements of $B$ are zero and we find that $A B A=A$. Thus $A$ is regular. Hence $M$ is a regular $r$-semigroup.

DEFINITION 2.3. Let $M$ be a r-semigroup. An element e $\varepsilon M$ is said to be an idempotent of $M$ if there exists an $\alpha \varepsilon \Gamma$ such that $e \propto=e$. In this case we shall say e is an a-idempotent.

DEFINITION 2.4. Let $M$ be a $\Gamma$-semigroup and $a \varepsilon M$. Let $b \varepsilon M$ and $\alpha, \beta \varepsilon \Gamma$. $b$ is said to be an $(\alpha, \beta)$ inverse of $a$ if $a=a a b \beta a$ and $b=b \beta a a b$. In this case we shall write $b \in v_{\alpha}^{\beta}(a)$.

DEFINITION 2.5. A regular $\Gamma$-semigroup $M$ is called an inverse $\Gamma$-semigroup if $\left|v_{\alpha}^{\beta}(a)\right|=1$, for all a $\varepsilon M$ and for all $\alpha, \beta \varepsilon \Gamma$, whenever $v_{\alpha}^{\beta}(a) \neq \phi$. That is, every element a of $M$ has a unique $(\alpha, \beta)$ inverse whenever the $(\alpha, \beta)$ inverse of a exists.

THEOREM 2.1. Let $M$ be a $\Gamma$-semigroup. $M$ is an inverse $\Gamma$-semigroup if and only if (i) $M$ is regular and (ii) if $e$ and $f$ are any two $\alpha$-idempotents of $M$ then $e$ of $=f \propto$, where $\alpha \in \Gamma$.

LEMMA 2.2. Let $M$ be a regular $\Gamma$-semigroup and let $M^{\prime}$ be a $\Gamma^{\prime}$-semigroup. Let $(f, g)$ be a homomorphism from $(M, \Gamma)$ onto $\left(M^{\prime}, \Gamma^{\prime}\right)$. Then $M^{\prime}$ is a regular $\Gamma^{\prime}$-semigroup.

3. ORTHODOX T-SEMIGROUP.

DEFINITION 3.1. A regular $\Gamma$-semigroup $M$ is called an orthodox $\Gamma$-semigroup if for $e$ an $\alpha$-idempotent and $f$ a $\beta$-idempotent then eof, $f \propto$ are $\beta$-idempotents and e $\beta f$, $f \beta e$ are $\alpha$-idempotents.

EXAMPLE 3.1. Let $A=\{1,2,3\}$ and $B=\{4,5\}$. $M$ denotes the set of all mappings from $A$ to $B$. Here members of $M$ will describe the images of the elements $1,2,3$. For example the map $1 \rightarrow 4,2 \rightarrow 5,3+4$ will be written as $(4,5,4)$ and $(4,4,5)$ denotes the map

$1 * 4,2 * 4,3+5$. Again a map from $B \rightarrow A$ will be in the same fashion. For example $(1,2)$ denotes $4 \rightarrow 1,5 \rightarrow 2$. Now, $M=\{(4,4,4),(4,4,5),(4,5,4),(4,5,5),(5,5,5),(5,4,5)$, $(5,4,4),(5,5,4)\}$. Let $\Gamma=\{(1,1),(1,2),(1,3),(2,1),(2,2),(3,3)\}$ be a set of some mappings from $B$ to $A$. Let $f, g \varepsilon M$ and $\alpha \varepsilon r$. Under the usual mapping composition, $f \circ$ is a mapping from $A$ to $B$ and hence $f \circ \varepsilon M$. Also, we can easily show that $(f \circ) B h=f \alpha(g \beta h)$, for all $f, g, h \varepsilon M$ and $\alpha, \beta \varepsilon r$. One can easily verify that $M$ is a regular $\Gamma$-semigroup. Here

$$
\begin{aligned}
& (4,4,4)(1,1)(4,4,4)(1,1)(4,4,4)=(4,4,4) \\
& (4,4,5)(1,3)(4,4,5)(1,3)(4,4,5)=(4,4,5) \\
& (4,5,4)(1,2)(4,5,4)(1,2)(4,5,4)=(4,5,4) \\
& (4,5,5)(1,2)(4,5,5)(1,2)(4,5,5)=(4,5,5) \\
& (5,5,5)(1,1)(5,5,5)(1,1)(5,5,5)=(5,5,5) \\
& (5,4,5)(1,2)(5,4,4)(1,2)(5,4,5)=(5,4,5) \\
& (5,4,4)(1,2)(5,4,5)(1,2)(5,4,4)=(5,4,4)
\end{aligned}
$$




$$
(5,5,4)(1,2)(5,4,5)(1,3)(5,5,4)=(5,5,4)
$$

Here $(4,4,5)$ is $(1,3)$ idempotent, and $(5,4,4)$ is $(2,1)$ idempotent, but $(4,4,5)(1,3)$ $(5,4,4)=(5,5,4)$ is not an idempotent. Hence this regular r-semigroup is not an orthodox $\Gamma$-semigroup.

EXAMPLE 3.2. Let $Q^{*}$ denote the set of all non-zero rational numbers. Let $\Gamma$ be the set of all positive integers. Let $a \in Q^{*}, \alpha \varepsilon \Gamma$ and $b \varepsilon Q^{*}$. a ob is defined by $|a|$ ab. For this operation $Q^{*}$ is a $\Gamma$-semigroup. Let $\frac{P}{q} \in Q^{*}$. Now

$\left|\frac{p}{q}\right||q|\left|\frac{1}{p}\right| 1 \frac{p}{q}=\frac{p}{q}$. Hence this is a regular $\Gamma$-semigroup. Here $\frac{1}{q},|q| \varepsilon \Gamma$ is a $|q|$ idempotent. These are the only idempotents of $Q^{*}$. Now $\frac{1}{q}|q| \frac{1}{p}$ is a $q$ p广idempotent. Hence $Q^{*}$ is an orthodox $\Gamma$-semigroup.

THEOREM 3.3. Every inverse $\Gamma$-semigroup is an orthodox $\Gamma$-semigroup.

PROOF. Let $M$ be an inverse $\Gamma$-semigroup. Let $e$ be a $\alpha$-idempotent and $f$ be a $\beta$-idempotent. Now eof $\varepsilon M$. Since $M$ is an inverse $\Gamma$-semigroup, let $x \in V_{\delta}^{\gamma}(e$ of $)$. Then e of $\delta x$ ye of $=e$ of, $x y e$ of $\delta x=x$. Let $g=f \delta x$ e of. Then $g$ ag $=g$. Also, let $h=f \delta x y$. Then, $f \delta x y e$ of $\beta f \delta x y e$ of $\delta x y e$ of $=f \delta x y e$ of $\delta x y e$ of $=f \delta x$ ye of $=g$. This shows that $g$ thag $=g$. Similarly hag $\beta h=h$. Hence $g \varepsilon_{\alpha}^{\beta}(h)$. Also e of $\varepsilon v_{\alpha}^{\beta}(h)$. Since $M$ is an inverse $r$-semigroup, $g=e$ of. Hence eof is a $\beta$-idempotent. Similarly we can show that $f \propto$ is $\beta$-idempotent, and both $e \beta f$ and $f \beta e$ are $\alpha$-idempotents.

EXAMPLE 3.4. In example 3.2 we have shown that $Q^{*}$ is an orthodox $\Gamma$-semigroup. Now $(1 / q) \varepsilon v_{p}^{1}(q / p)$. Also $(-1 / q) \in v_{p}^{1}(q / p)$. Hence $Q^{*}$ is not an inverse $r$-semigroup.

THEOREM 3.5. A regular $\Gamma$-semigroup $M$ is an orthodox $\Gamma$-semigroup if an only if for any $\alpha$-idempotent e $\varepsilon M$, where $\alpha \varepsilon r$, if $\mathrm{v}_{\alpha}^{\beta}(\mathrm{e}) \neq \phi$ and $\mathrm{v}_{\beta}^{\alpha}(e) \neq \phi$, then each member of $v_{\alpha}^{\beta}(e)$ and $v_{\beta}^{\alpha}(e)$ is a $\beta$-idempotent.

PROOF. Suppose $M$ is an orthodox $\Gamma$-semigroup. Let $e$ be an a-idempotent of $M$ and let $x \in v_{\alpha}^{\beta}(e)$. Then $e \alpha x \beta=e$ and $x \beta e \alpha x=x$. Now e $\alpha x$ is a $\beta$-idempotent and $x \beta e$ is an $\alpha$-idempotent. Then $x=(x \beta e) \alpha(e \alpha x)$ is a $\beta$-idempotent. Next let $y \in v_{\beta}^{\alpha}(e)$. Then $e \beta y \propto e=e$ and $y \propto \beta y=y$. Now $y \propto$ is a $\beta$-idempotent and $e \beta y$ is an o-idempotent. Then $y=(y \propto) \alpha(e \beta y)$ is a $\beta$-idempotent. Conversely suppose that M satisfies the given conditions. Let $e$ be an $\alpha$-idempotent and $f$ be a $\beta$-idempotent. Consider $e$ of. Now e of $\varepsilon M$, and since $M$ is regular there exists $x \in M$ and $\gamma, \delta \varepsilon \Gamma$ such that $e$ of $y$ \&e of $=e$ of and $x$ \&e of $\gamma=x$. Let $g=f \gamma x$. Then $g \propto g=f \gamma(x \delta e$ of $\gamma x) \delta e=f \gamma x \delta=g$. Now, e of $\beta f \gamma x \delta e$ of $=e$ of $\gamma x \delta e$ of $=e$ of and $f \gamma x \delta e \alpha$ of $\beta f \gamma x \delta=f \gamma x \delta e$ of $\gamma x \delta e=f \gamma x \delta$. Hence e of $\varepsilon v_{\alpha}^{\beta}(f y x \delta)$. Then by the given condition eof is $\beta$-idempotent. Dually we can prove that $f \propto e$ is $\beta$-idempotent. Similarly, it is easy to see that $e \beta f$ and $f \beta e$ are $\alpha$-idempotents.

THEOREM 3.6. A regular $\Gamma$-semigroup $M$ is an orthodox $\Gamma$-semigroup if and only if for $a, b \varepsilon M, \alpha_{1}, \alpha_{2}, \beta_{1}, \beta_{2} \varepsilon \Gamma, a^{\prime} \varepsilon v_{\alpha_{1}}^{\alpha}(a)$, and $b^{\prime} \varepsilon v_{\beta_{1}}^{\beta_{2}}(b)$ we have $b^{\prime} \beta_{2} a^{\prime} \in v_{\beta_{1}}^{\alpha_{2}}\left(a \alpha_{1} b\right)$ and $b^{\prime} \alpha_{1} a^{\prime} \in v_{\beta_{1}}^{\alpha_{2}}\left(a \beta_{2} b\right)$. 
PROOF. Let us assume that $M$ is an orthodox $\Gamma$-semigroup. Let $a^{\prime} \varepsilon v_{\alpha_{1}}^{\alpha_{2}}(a)$ and $b^{\prime} \varepsilon v_{\beta_{1}}^{\beta_{2}}(b)$. Then $a \alpha_{1} a^{\prime} \alpha_{2} a=a, a^{\prime} \alpha_{2} a_{1} a^{\prime} a^{\prime}=a^{\prime}, b \beta_{1} b^{\prime} \beta_{2} b=b$, and $b^{\prime} \beta_{2} b \beta_{1} b^{\prime}=b^{\prime}$.

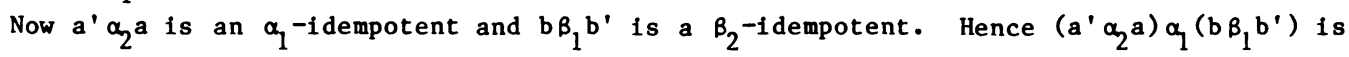
$a \beta_{2}$-idempotent, and $\left(b \beta_{1} b^{\prime}\right) \beta_{2}\left(a^{\prime} \alpha_{2} a^{a}\right)$ is an $\alpha_{1}$-idempotent. $\quad\left(a^{\prime} \alpha_{2} a\right) \beta_{2}\left(b \beta_{1} b^{\prime}\right)$ is an $\alpha_{1}$-idempotent and $\left(b \beta_{1} b^{\prime}\right) \alpha_{1}\left(a^{\prime} \alpha_{2} a\right)$ is a $\beta_{2}$-idempotent.

$$
\begin{aligned}
& a \alpha_{1} b^{b} b^{\prime} \beta_{2} a^{\prime} \alpha_{2} a \alpha_{1} b=a \alpha_{1} a^{\prime} \alpha_{2} a \alpha_{1} b \beta_{1} b^{\prime} \beta_{2} a^{\prime} \alpha_{2} a \alpha_{1} b \beta_{1} b^{\prime} \beta_{2} b \\
& =a \alpha_{1} a^{\prime} \alpha_{2} a \alpha_{1} b \beta_{1} b^{\prime} \beta_{2} b^{\prime} \text { (since } a^{\prime} \alpha_{2} a \alpha_{1} b \beta_{1} b^{\prime} \text { is } \beta_{2} \text {-idempotent) } \\
& =a \alpha_{1} b \text {. } \\
& b^{\prime} \beta_{2} a^{\prime} \alpha_{2} a \alpha_{1} b^{b} b^{\prime} \beta_{2} a^{\prime}=b^{\prime} \beta_{2} b \beta_{1} b^{\prime} \beta_{2} a^{\prime} \alpha_{2} a \alpha_{1} b_{1} b^{\prime} \beta_{2} a^{\prime} \alpha_{2} a \alpha_{1} a^{\prime} \\
& =b^{\prime} \beta_{2} b^{b} b_{1} \beta_{2} a^{\prime} \alpha_{2} a \alpha_{1} a^{\prime} \text { (since } b \beta_{1} b^{\prime} \beta_{2} a^{\prime} \alpha_{2} a^{a} \text { is } \alpha_{1} \text {-idempotent) } \\
& =b^{\prime} B_{2} a^{\prime} \text {. }
\end{aligned}
$$

Hence $b^{\prime} \beta_{2} a^{\prime} \varepsilon v_{\beta_{1}}^{\alpha_{2}}\left(a \alpha_{1} b\right)$. Similarly it can be shown that $b^{\prime} \alpha_{1} a^{\prime} \varepsilon v_{\beta_{1}}^{\alpha_{2}}\left(a \beta_{2} b\right)$. Conversely, assume that the given conditions hold in $M$. Let $e$ be an $\alpha$-idempotent and $f$ be a $\beta$-idempotent of $M$. Now $f \in v_{\beta}^{\beta}(f)$ and $e \in v_{\alpha}^{\alpha}(e)$. Then by the given conditions

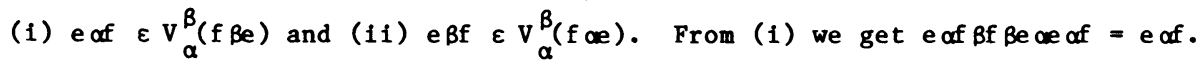

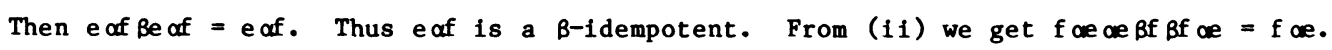

Then $f \propto \beta f \propto=f \propto$. So, $f \propto$ is $\beta$-idempotent. Again

e $\varepsilon v_{\alpha}^{\alpha}(e)$ and $f \in v_{\beta}^{\beta}(f)$. Then by the given conditions we get (iii) $f \beta e \varepsilon V_{\beta}^{\alpha}$ (e of )

and (iv) $f \propto \varepsilon v_{\beta}^{\alpha}(e \beta f)$. From (iii) we get $f \beta e \propto \alpha \beta f \beta e=f \beta e$. Then $f \beta e$ of $\beta e=f \beta e$.

Hence $f \beta e$ is $\alpha$-idempotent. From (iv) we get e $\beta f \beta f \propto e \propto \beta f=e \beta f$. So, e $\beta f \propto \beta f=e \beta f$.

Thus e $\beta$ is $\alpha$-idempotent. Hence $M$ is an orthodox $r$-semigroup.

THEOREM 3.7. A regular $\Gamma$-semigroup $M$ is an orthodox $\Gamma$-semigroup if and only if for $a, b \in M, v_{\alpha}^{\beta}(a) \cap v_{\alpha}^{\beta}(b) \neq \phi$ for some $\alpha, \beta \varepsilon r$. This implies that $v_{\gamma}^{\delta}(a)=v_{\gamma}^{\delta}(b)$ for all $\gamma, \delta \in \Gamma$.

PROOF. Suppose $M$ is an orthodox $\Gamma$-semigroup. For $a, b \in M$, let there exist $\alpha, \beta \in \Gamma$ such that $v_{\alpha}^{\beta}(a) \cap v_{\alpha}^{\beta}(b) \neq \phi$. Let $\gamma, \delta \in \Gamma$. First let us show that $v_{\gamma}^{\delta}(a) \subset v_{\gamma}^{\delta}(b)$. Let $a^{\prime} \varepsilon v_{\alpha}^{\beta}(a) \cap v_{\alpha}^{\beta}(b)$, and $a^{*} \varepsilon v_{\gamma}^{\delta}(a)$. Then $a a^{\prime} \beta a=a$, $a^{\prime} \beta a c a^{\prime}=a^{\prime}, b a^{\prime} \beta b=b, a^{\prime} \beta b \alpha a^{\prime}=a^{\prime}, \quad a y^{\star} \delta a=a, a^{\star} \delta a a^{\star}=a^{\star}$. We can easily show that 


$$
(a * \delta a) \alpha\left(a^{\prime} \beta b\right) R(a * \delta a)
$$

Now $a^{\star} \delta a$ is $\gamma$-idempotent, and $a^{\prime} \beta b$ is $\alpha$-idempotent. Hence $\left(a^{\star} \delta a\right) \alpha\left(a^{\prime} \beta b\right)$ is

r-idempotent. Then from (3.1) we get

$$
(a * \delta a) \alpha\left(a^{\prime} B b\right) \gamma(a * \delta a)=a * \delta a \text {. }
$$

Now $a^{\prime} \beta a=a^{\prime} \beta a \gamma a \star \delta a=a^{\prime} \beta a \gamma a * \delta a \alpha a^{\prime} \beta b \gamma a * \delta a=a^{\prime} \beta a \alpha a{ }^{\prime} \beta b \gamma a * \delta a=a^{\prime} \beta b \gamma a * \delta a$.

Hence $b_{c a}^{\prime} B a=b c a^{\prime} B b \gamma a * \delta a=b \gamma a * \delta a$.

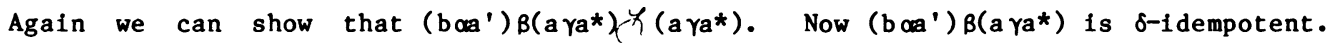

Hence $\left(a \gamma a^{*}\right) \delta\left(b c a^{\prime}\right) B\left(a \gamma a^{*}\right)=a \gamma a^{*}$.

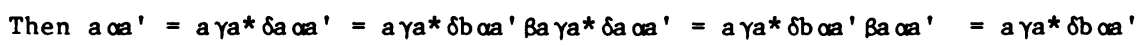

$a \propto a^{\prime} \beta b=a b^{\prime}{ }^{*} \delta b \propto a^{\prime} \beta b=a b^{\prime}{ }^{*} \delta b$.

Now, $b \gamma a * \delta b=b \gamma a * \delta a \gamma a * \delta b$

$=b \gamma a * \delta a \alpha a^{\prime} \beta b \quad$ by $(3.4)$

$=b \alpha a^{\prime} B a \alpha a^{\prime} B b$ by (3.3)

$=b^{\prime} a^{\prime} B b=b$

and $a * \delta b \gamma a *=a * \delta a \gamma a * \delta b \gamma a * \delta a \gamma a *$

$=a * \delta a \gamma a * \delta b \propto a a^{\prime}$ Ba $\gamma a *$ by (3.3)

$=a * \delta a \alpha a^{\prime} B b \alpha a^{\prime} B a \gamma a *$ by (3.4)

$=a * \delta a \propto a^{\prime}$ Ba $\gamma a *=a * \delta a \gamma a *=a *$.

Hence $a * \varepsilon v_{\gamma}^{\delta}(b)$. Thus $v_{\gamma}^{\delta}(a) \subset v_{\gamma}^{\delta}(b)$. Similarly $v_{\gamma}^{\delta}(b) \subset v_{\gamma}^{\delta}(a)$. Thus $v_{\gamma}^{\delta}(a)=v_{\gamma}^{\delta}(b)$. Conversely, assume that the given condition holds in M. Let $e$ be $\alpha$-idempotent and $f$ be $B$-idempotent. Consider eof. Since $M$ is regular, there exists $\gamma, \delta \varepsilon \Gamma$ and $x \in M$ such that $e$ of $\gamma x \delta e$ of $=e$ of and $x \delta e$ of $\gamma x=x$. Let $g=f \gamma x \delta$. Then $g$ og $=g$. Hence $f \gamma x \& \varepsilon v_{\alpha}^{\alpha}(f y x \delta)$. Let $h=e$ of $\gamma x \delta$. Then $h$ oh $=h$. Also, $f \gamma x \delta e v_{\alpha}^{\alpha}(e$ of $\gamma x \delta)$. Hence $v_{\alpha}^{\alpha}(g) \cap v_{\alpha}^{\alpha}(h) \neq \phi$. Then $v_{\alpha}^{\theta}(g)=v_{\alpha}^{\theta}(h)$ for any $\theta \varepsilon \Gamma$. But e of $\beta f \gamma x \delta \alpha$ of $=$ e of and $f \gamma x \delta a$ of $\beta f \gamma x \delta e=f \gamma x \delta e$. Hence e of $\varepsilon v_{\alpha}^{\beta}(f \gamma x \delta e)$. Then e of $\varepsilon v_{\alpha}^{\beta}(e$ of $\gamma x \delta)$. This implies that e of $\beta e$ of $\gamma x \delta e$ of $=e$ of. So e of $B e$ of $=e$ of. Hence e of is $\beta$-idempotent. Similarly, it can be proved that $f \propto$ is a $\beta$-idempotent and both $e \beta f$ and $f B e$ are a-idempotents. Let $M$ be a regular $\Gamma$-semigroup and $a, b \varepsilon M, a^{\prime} \varepsilon V_{\alpha}^{\beta}(a)$, and $b^{\prime} \varepsilon v_{\gamma}^{\delta}(b)$. Then $e=a^{\prime} B a$ is $\alpha$-idempotent and $f=b_{\gamma} b^{\prime}$ is $\delta$-idempotent. Let $\theta \varepsilon r$. Suppose $x \in v_{\alpha_{1}}^{\beta_{1}}(e \theta f)$. Then e $\theta f=$ e $\theta f \alpha_{1} x \beta_{1}$ e $\theta f$ and $x=x \beta_{1}$ e $\theta f \alpha_{1} x$.

Let $g=f \alpha_{1} \times \beta_{1} e$. Now $g \theta g=f \alpha_{1} \times \beta_{1} e \theta f \alpha_{1} \times \beta_{1} e=f \alpha_{1} \times \beta_{1} e=g$. Hence $g$ is $\theta$-idempotent. 
Also $g \propto=g=f \delta g$ and $e \theta g \theta f=e \theta f$. Now,

$$
\begin{aligned}
(a \theta b) \gamma\left(b^{\prime} \delta g \alpha a^{\prime}\right) \beta(a \theta b) & =a \theta\left(b \gamma b^{\prime}\right) \delta g \alpha\left(a^{\prime} \beta a\right) \theta b=a \theta f \delta g \propto b \\
& =a \theta f \delta \theta \theta=a \theta g \theta b=a \alpha a^{\prime} \beta a \theta \theta b \gamma b^{\prime} \delta b \\
& =a \propto \theta g \theta \delta b=a \propto \theta f \delta b=a \theta b .
\end{aligned}
$$

Similarly we can show that $\left(\beta^{\prime} \delta g \alpha a^{\prime}\right) \beta(a \theta b) \gamma\left(b^{\prime} \delta g \alpha a^{\prime}\right)=b^{\prime} \delta g \alpha a^{\prime}$. Hence

$b^{\prime} \delta g \propto a^{\prime} \varepsilon V_{\gamma}^{\beta}(a \theta b)$. Thus we have the following lemma.

LEMMA 3.8. Let $M$ be a regular $\Gamma$-semigroup and $a, b \varepsilon M$. If $a^{\prime} \varepsilon V_{\alpha}^{\beta}(a)$, $b^{\prime} \varepsilon V_{\gamma}^{\delta}(b)$ and $\theta \varepsilon r$, then there exists a $\theta$-idempotent $g \varepsilon M$ and $b^{\prime} \delta g a a^{\prime} \varepsilon V_{\gamma}^{\beta}(a \theta b)$.

4. INVERSE T-SEMIGROUP CONGRUENCE.

DEFINITION 4.1. Let $M$ be a $\Gamma$-semigroup. A congruence on $M$ is defined as an equivalence relation $\rho$ on the set $M$ stable under left and right $\Gamma$-operations. That is, for every $a, b, c \varepsilon M,(a, b) \varepsilon \rho$ implies $(c a a, c a b) \varepsilon \rho$ and $(a a c, b a c) \varepsilon \rho$ for all $\alpha \varepsilon \Gamma$. A left (right) congruence on $M$ is an equivalence relation on $M$, stable under left (right) r-operation.

Let $M$ be a $\Gamma$-semigroup. Let $\rho$ be a congruence on $M$. We define $(a \rho) \alpha(b \rho)=(a a b) \rho$ for all a $\rho, b \rho \varepsilon M / \rho$ and for all $\alpha \varepsilon \Gamma$. It can easily be seen that the definition is well defined and $M / \rho$ is a $\Gamma$-semigroup. Let us now characterize the minimum inverse $\Gamma$-semigroup congruence on an orthodox $\Gamma$-semigroup.

DEFINITION 4.2. Let $M$ be an orthodox $\Gamma$-semigroup. A congruence $\rho$ on $M$ will be called an inverse $\Gamma$-semigroup congruence if $M / \rho$ is an inverse $\Gamma$-semigroup.

THEOREM 4.1. Let $M$ be an orthodox $\Gamma$-semigroup. Then the relation $\rho$ defined by $\rho=\left\{(a, b) \varepsilon M x M: V_{\alpha}^{\beta}(a)=v_{\alpha}^{\beta}(b)\right.$ for all $\left.\alpha, \beta \varepsilon \Gamma\right\}$ is the minimum inverse $\Gamma$-semigroup congruence on $M$.

PROOF. From the definition of $\rho$ it is clear that $\rho$ is an equivalence relation. To prove that $\rho$ is a congruence relation, assume that $(a, b) \varepsilon \rho, c \varepsilon M$ and $\theta \varepsilon \Gamma$. Then $v_{\alpha}^{\beta}(a)=v_{\alpha}^{\beta}(b)$ for all $\alpha, \beta \varepsilon \Gamma$. Hence there exists $\alpha, \beta \varepsilon \Gamma$ such that $\mathrm{v}_{\alpha}^{\beta}(\mathrm{a})=\mathrm{v}_{\alpha}^{\beta}(\mathrm{b}) \neq \phi$. Let $\mathrm{a} * \varepsilon \mathrm{V}_{\alpha}^{\beta}(\mathrm{a})=\mathrm{v}_{\alpha}^{\beta}(\mathrm{b}), \mathrm{c}^{*} \varepsilon \mathrm{v}_{\gamma}^{\delta}(\mathrm{c})$. Then by Lemma 3.8 $c * \delta g \alpha * \varepsilon V_{\gamma}^{\beta}(a \theta c)$ and $c * \delta g \alpha a * \varepsilon V_{\gamma}^{\beta}(b \theta c)$, for some $\theta$-idempotent $g=g \theta g \varepsilon M$. Hence $v_{\gamma}^{\beta}(a \theta c) \cap v_{\gamma}^{\beta}(b \theta c) \neq \phi$. Then by Theorem 3.7, $v_{\alpha}^{\beta}(a \theta c)=v_{\alpha}^{\beta}(b \theta c)$ for a11 $\alpha, \beta \varepsilon \Gamma$, so that 
$(a \theta c, b \theta c) \varepsilon \rho$. Similarly we can show that $(c \theta a, c \theta b) \varepsilon \rho$. Hence $\rho$ is a congruence on $M$. Suppose now $e=e \propto$ and $f=f$ of are two idempotents of $M$. Then $e$ of and $f \propto e$ are $\alpha$-idempotents and eof $\varepsilon v_{\alpha}^{\alpha}(e$ of $)$ and eof $\varepsilon v_{\alpha}^{\alpha}(f \propto e)$. Hence $v_{\alpha}^{\alpha}(e \alpha f) \cap v_{\alpha}^{\alpha}(f \propto) \neq \phi$. Consequently $v_{\gamma}^{\delta}(e a f)=v_{\gamma}^{\delta}(f \propto e)$ for all $\gamma, \delta \varepsilon \Gamma$. Thus we find that (eaf, fœ) $\varepsilon \rho$. Hence from Theorem 2.1 and Lemma 2.2 we $f$ ind that $M / \rho$ is an inverse $\Gamma$-semigroup. Finally, suppose that $\rho_{1}$ is a congruence on $M$ such that $M / \rho_{1}$ is an inverse r-semigroup. If $(a, b) \varepsilon \rho$ then $v_{\alpha}^{\beta}(a)=v_{\alpha}^{\beta}(b)$ for all $\alpha, \beta \varepsilon \Gamma$. There exist $x \in M$ and $\alpha, \beta \varepsilon \Gamma$ such that $a \propto \beta a=a, \quad x \beta a \propto x=x, \quad b \propto \alpha \beta b=b$ and $x \beta b \propto x=x$.

Then $\left(a \rho_{1}\right) \alpha\left(x \rho_{1}\right) B\left(a \rho_{1}\right)=a \rho_{1},\left(x \rho_{1}\right) \beta\left(a \rho_{1}\right) \alpha\left(x \rho_{1}\right)=x \rho_{1},\left(b \rho_{1}\right) \alpha\left(x p_{1}\right) \beta\left(b \rho_{1}\right)=b \rho_{1}$, $\left(x \rho_{1}\right) \beta\left(b \rho_{1}\right) \alpha\left(x \rho_{1}\right)=x \rho_{1}$. Hence $a \rho_{1}, b \rho_{1} \varepsilon v_{\beta}^{\alpha}\left(x \rho_{1}\right)$. But $M / \rho_{1}$ is an inverse r-semigroup. Hence $\left|v_{\beta}^{\alpha}\left(x \rho_{1}\right)\right|=1$. Then $a \rho_{1}=b \rho_{1}$, so that $(a, b) \varepsilon \rho_{1}$. Hence $\rho \subset \rho_{1}$. This completes the proof.

\section{REFERENCES}

1. SAHA, N.K., On T-semigroup II, Bul1. Cal. Math. Soc. 79 (1987), 331-335.

2. SEN, M.K. and SAHA, N.K., On I-semigroup I, Bu1l. Cal. Math. Soc. 78(1986), 180186.

3. SAHA, N.K., On Г-semigroup III, Bul1. Cal. Math. Soc. 80 (1988), 1-12.

4. SAHA, N.K. and SETH, A., Inverse $\Gamma$-semigroup. Accepted for publication in J. of Pure Math. Calcutta University.

5. HALL, T.E., On regular semigroups whose idempotents form a subsemigroup, Bul1. Australian Math. Soc. I (1967).

6. YAMADA, M., Regular semigroups whose idempotents satisfy permutation identities. Pacific J. Math. 21(2) (1967), 371-392. 


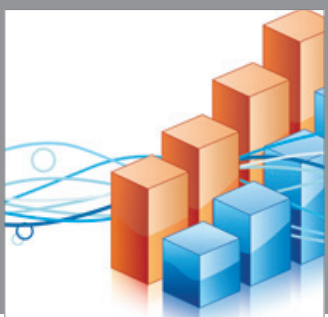

Advances in

Operations Research

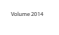

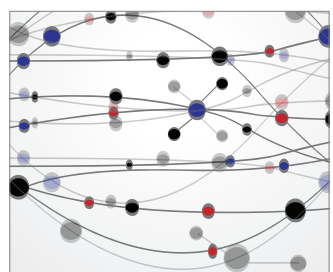

\section{The Scientific} World Journal
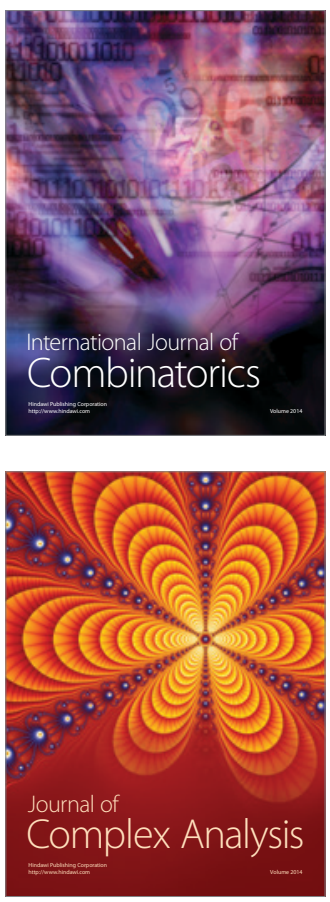

International Journal of

Mathematics and

Mathematical

Sciences
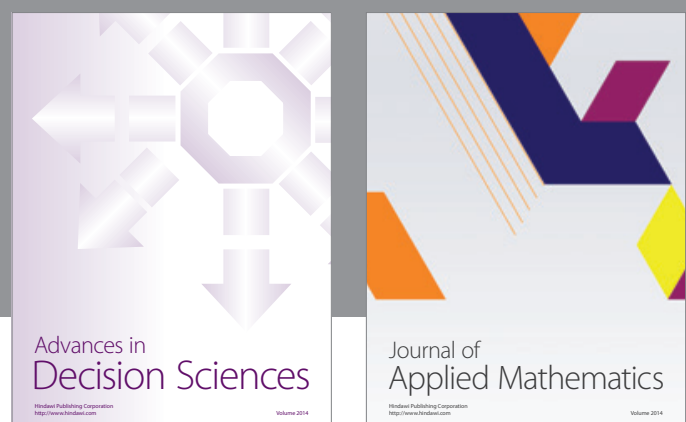

Journal of

Applied Mathematics
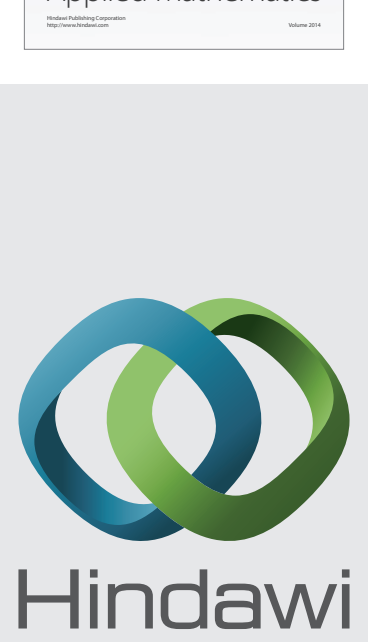

Submit your manuscripts at http://www.hindawi.com
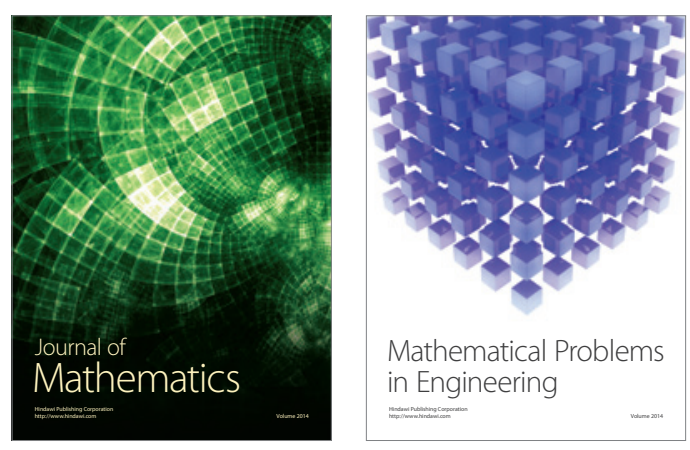

Mathematical Problems in Engineering
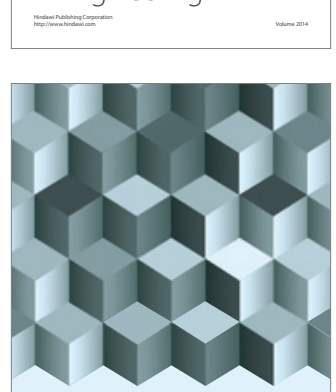

Journal of

Function Spaces
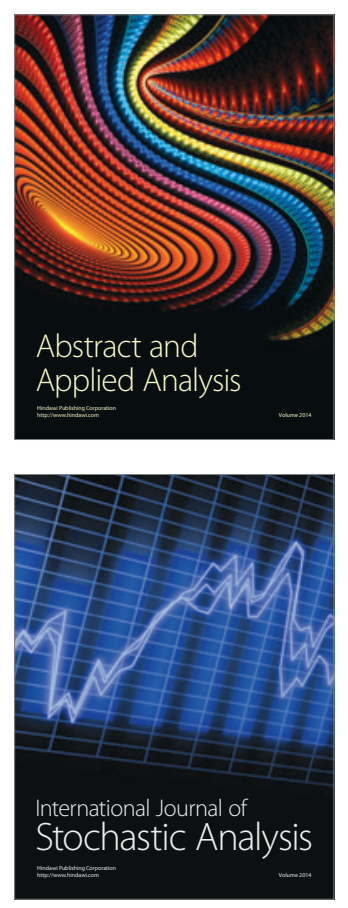

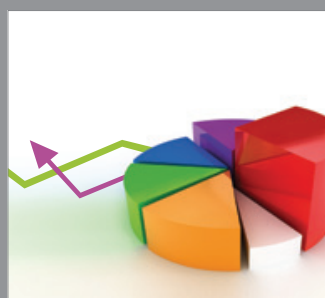

ournal of

Probability and Statistics

Promensencen
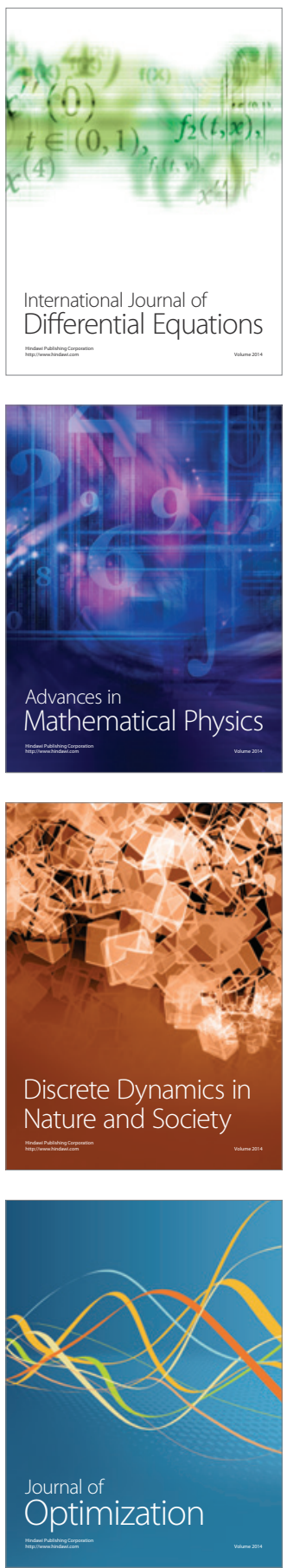